\title{
STOCHASTIC MODELS FOR OPTICALLY TRAPPED NANOWIRES
}

\author{
IGNACIO ORTEGA-PIWONKA
}

(Received 17 October 2017; first published online 30 May 2018)

2010 Mathematics subject classification: primary 37H10, secondary 82C31, 82D80.

Keywords and phrases: complex systems, optical physics, stochastic differential equations.

In many physical systems noise plays a minor role, as a perturbation to deterministic motion; or a major role, in suppressing a deterministic signal. In this thesis we have considered a system in which noise plays a fundamental role in the observed dynamics. The particular system that we study consists of a nanowire that is suspended in a fluid, trapped by optical tweezers and subject to noise from collisions with the fluid particles. The motion of the nanowire is overdamped but experiments reveal a persistent cyclical motion. We have developed theoretical models, based on overdamped Langevin equations, which can accurately describe the observed behaviour of the nanowire. In these models the cyclical motion occurs as a result of coupling between the noise and the optical force, when the latter is nonconservative. The models may thus be used to probe the presence of nonconservative forces in optical tweezers experiments.

The solutions of overdamped Langevin equations are single realisations of a stochastic trajectory of a particle in a fluid, subject to random fluctuations and a force field. The Fokker-Planck equation is an alternative formulation, whose solution describes the evolution of the probability density for the position of the particle. The Fokker-Planck equation can be derived from a microscopic description in terms of random walks. In order to consider more exotic fluids, including viscoelastic fluids, we have derived a fractional Fokker-Planck equation from a mesoscopic description involving continuous-time random walks with arbitrary initial conditions. Our derived model will facilitate the investigation of the motion of particles, such as nanowires, suspended in exotic fluids and trapped by optical tweezers.

An intriguing aspect of the work in this thesis is that noise can play a constructive role in producing ordered behaviour.

Thesis submitted to the University of New South Wales in February 2017; degree approved on 26 July 2017; supervisor Bruce Henry, co-supervisors Christopher Angstmann and Peter Reece.

(C) 2018 Australian Mathematical Publishing Association Inc. 
IGNACIO ORTEGA-PIWONKA, School of Mathematics and Statistics, University of New South Wales, NSW 2052, Australia

e-mail: i.ortegapiwonka@unswalumni.com 